\title{
Benign Genitourinary System Neoplasm
}

National Cancer Institute

\section{Source}

National Cancer Institute. Benign Genitourinary System Neoplasm. NCI Thesaurus. Code C156483.

A non-metastasizing neoplasm that arises from the genitourinary system. 